\title{
COMPACT FUNCTORS AND THEIR DUALS IN CATEGORIES OF BANACH SPACES
}

\author{
BY \\ KENNETH L. POTHOVEN( $\left.{ }^{1}\right)$
}

\begin{abstract}
In a recent paper, B. S. Mityagin and A. S. Shvarts list many problems concerning functors and dual functors in categories of Banach spaces. Included in these problems is the question: What properties characterize compact functors? The purpose of this paper is to give partial answers to that question. Partial characterizations are given in terms of what are called Fredholm functors and finite rank functors. Affirmative answers are also given to two other questions of Mityagin and Shvarts. They are (1) If a functor is compact, is its dual compact? (2) If a natural transformation is compact, is its dual compact?
\end{abstract}

1. Preliminaries. The letter $\boldsymbol{B}$ will denote the category in which the objects are Banach spaces $A, B, C, X$, or $Y$-real or complex, but not both. The scalar field is denoted by $I$. The morphism set $\boldsymbol{B}(A, B)$ corresponding to an ordered pair $(A, B)$ of objects is the Banach space of all continuous linear mappings $f, g, h$, or $k$ from $A$ to $B$. The norm of $f$ in $B(A, B)$ is given by $|f|=\sup _{|a| \leqq 1}|f(a)|$.

In addition to the usual definition of a (covariant) functor [4, p. 49], all functors $F: B \rightarrow B$ are assumed to satisfy the condition that for each $A$ and $B$ in $B$, the induced function $F_{A B}: B(A, B) \rightarrow B(F(A), F(B))$ is a linear contraction (that is, $|F(f)| \leqq|f|)$. Let $F$ and $G$ be functors from $B$ to $B$. In addition to the usual definition of a natural transformation [4, p. 59], all natural transformations $\eta: F \rightarrow G$ must satisfy the condition that the "norm" of $\eta-|\eta|=\sup \left\{\left|\eta_{X}\right|: X\right.$ in $\left.\boldsymbol{B}\right\}$-is finite, where $\eta_{X}: F X \rightarrow G X$ is the morphism assigned by $\eta$ to the object $X$.

If $F$ and $G$ are two functors from $B$ to $B,(F \rightarrow G)$ will denote the class of all natural transformations from $F$ to $G$. When $(F \rightarrow G)$ is a set, it is a Banach space with the norm defined above.

Let $\eta: F \rightarrow G$ be a natural transformation. If for each $X$ in $B, \eta_{X}: F X \rightarrow G X$ is an isomorphism (one-to-one and onto), then $\eta$ is called a natural isomorphism.

Presented to the Society, January 24, 1970 under the title Some results on compact functors and a characterization of the space $L_{1}(\mu)$; received by the editors January 19, 1970 and, in revised form, May 22, 1970.

AMS 1969 subject classifications. Primary 0810, 4745; Secondary 4610.

Key words and phrases. Compact functors, categories of Banach spaces, projective tensor product, weakly compact natural transformations, Fredholm functor, finite rank functor, approximation property.

(1) These results are part of those contained in the author's doctoral dissertation written at Western Michigan University under the direction of Professor Kung-Wei Yang.

Copyright (C) 1971, American Mathematical Society 
If for each $X$ in $B, \eta_{X}: F X \rightarrow G X$ is an equivalence (isometric isomorphism), then $F$ and $G$ are called naturally equivalent.

Two functors that will be prominent in the following discussion are the hom and tensor functors, designated for an $X$ in $B$ by $\Omega_{X}$ and $\Sigma_{X}$ respectively. For each $X$ in $B, \Omega_{X}$ is defined by the following assignments:

(1) If $A$ is in $B, \Omega_{X}(A)=B(X, A)$.

(2) If $f: A \rightarrow B$ is a morphism in $B$, then $\Omega_{X}(f): \Omega_{X}(A) \rightarrow \Omega_{X}(B)$ is given by $\Omega_{X}(f)(g)=f \circ g$ for $g \in \boldsymbol{B}(X, A)$.

For each $X$ in $B$, the functor $\Sigma_{X}$ is given by the following assignments:

(1) If $A$ is in $B, \Sigma_{X}(A)=X \hat{\otimes} A$, the completed projective tensor product. (The norm in $X \hat{\otimes} A$ is the greatest crossnorm of Schatten [8, p. 206].)

(2) If $f: A \rightarrow B$ is in $B, \Sigma_{X}(f): X \widehat{\otimes} A \rightarrow X \widehat{\otimes} B$ is the morphism $i_{X} \hat{\otimes} f$ which is the continuous extension of the morphism $i_{X} \otimes f: X \otimes A \rightarrow X \hat{\otimes} B$ given by the rule $i_{X} \otimes f\left(\sum x_{i} \otimes a_{i}\right)=\sum x_{i} \otimes f\left(a_{i}\right)$.

Definition 1.1. Let $f: \boldsymbol{B} \rightarrow \boldsymbol{B}$ be a functor. The dual functor $D F: \boldsymbol{B} \rightarrow \boldsymbol{B}$ to $F$ is the functor given by the following assignments:

(1) If $X$ is in $B, D F(X)=\left(F \rightarrow \Sigma_{X}\right)$. (It can be deduced from (2.2) that this is a set.)

(2) If $f: X \rightarrow Y$ is a morphism in $B, D F(f): D F(X) \rightarrow D F(Y)$ is the morphism given by the equation

$$
(D F(f)(\tau))_{A}=\left(f \hat{\otimes} i_{A}\right) \circ \tau_{A} \quad \text { for } \tau \in D F(X) \text { and } A \text { in } \boldsymbol{B} .
$$

Many examples of dual functors are given in [5]. There it is also proved that $D \Sigma_{X}$ is naturally equivalent to $\Omega_{X}$ for each Banach space $X$, and $D \Omega_{X}$ is naturally equivalent to $\Sigma_{X}$ for each Banach space $X$. It can easily be verified that $D I_{B}$ is naturally equivalent to $I_{B}$, the identity functor of $\boldsymbol{B}$.

Definition 1.2. Let $\tau: F \rightarrow G$ be a natural transformation from functor $F$ to $G$. The dual transformation $D \tau: D G \rightarrow D F$ to $\tau$ is the transformation which assigns to each object $X$ in $B$ in the morphism $(D \tau)_{X}: D G(X) \rightarrow D F(X)$ given by

$$
\left((D \tau)_{X} \eta\right)_{A}=\eta_{A} \circ \tau_{A} \text { for } \eta \in D G(X) \text { and } A \text { in } B \text {. }
$$

2. Compact functors, compact transformations, and their duals. This section is devoted to proving that the duals of compact functors and compact transformations are compact.

Definition 2.1. Let $\tau: F \rightarrow G$ be a natural transformation. To each morphism $f: X \rightarrow Y$ in $\boldsymbol{B}$ associate the morphism $\tau(f)$ defined as $\tau_{Y} \circ F(f)$ or its equal $G(f) \circ \tau_{X}: F X \rightarrow G Y$. The transformation $\tau$ is compact if whenever $f: X \rightarrow Y$ is compact (see [1, p. 485]), $\tau(f)$ is also a compact operator. A functor $F: B \rightarrow B$ is a compact functor if the identity natural transformation $\iota_{F}: F \rightarrow F$ is compact. Similarly the concepts of weakly compact, Fredholm (see after (3.1)), epimorphic, and monomorphic natural transformations and functors are defined. Thus for example, $\tau$ is a monomorphic transformation if whenever $f$ is a monomorphism, $\tau(f)$ is a monomorphism. 
For each $A$ in $B$, let $A^{*}$ be the conjugate (dual) space [1, p. 61]. Corresponding to each functor $F: B \rightarrow B$ is another functor $F^{\#}: \boldsymbol{B} \rightarrow \boldsymbol{B}$ given by the assignments:

(1) If $A$ is in $B, F^{\#}(A)=\left(F\left(A^{*}\right)\right)^{*}$.

(2) If $f: A \rightarrow B$ is a morphism in $B, F^{\#}(f)=\left(F\left(f^{*}\right)\right)^{*}:\left(F\left(A^{*}\right)\right)^{*} \rightarrow\left(F\left(B^{*}\right)\right)^{*}$ the adjoint of $F\left(f^{*}\right): F\left(B^{*}\right) \rightarrow F\left(A^{*}\right)$.

For each $A$ in $B$, let $\operatorname{Tr}: A^{*} \widehat{\otimes} A \rightarrow I$ be the continuous linear operator which is the unique extension of the map from $A^{*} \otimes A \rightarrow I$ given by $\sum_{i=1}^{n} a_{i}^{*} \otimes a_{i} \rightarrow$ $\sum_{i=1}^{n} a_{i}^{*}\left(a_{i}\right)$. (It can be checked that $|\operatorname{Tr}|=1$.) Also for each $A$ in $B$, define the continuous linear mapping $\lambda_{A}: D F(A) \rightarrow\left(F\left(A^{*}\right)\right)^{*}$ by the rule $\lambda_{A}(\theta)=\operatorname{Tr} \circ \theta_{A^{*}}$ for $\theta \in D F(A)$. This is shown by the following commutative diagram.

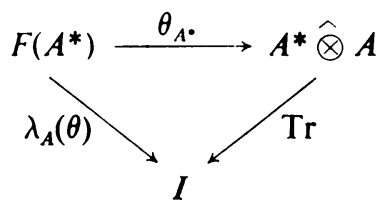

Since $\left|\lambda_{A}(\theta)\right|=\left|\operatorname{Tr} \circ \theta_{A^{*}}\right| \leqq\left|\theta_{A^{*}}\right| \leqq|\theta|,\left|\lambda_{A}\right| \leqq 1$.

LEMMA 2.2. For each functor $F: B \rightarrow B$, there is a natural transformation $\lambda: D F$ $\rightarrow F^{\#}$ where, for each $A$ in $B, \lambda_{A}$ is the morphism given above. Moreover, for each $A$ in $\boldsymbol{B}, \lambda_{A}$ is an isometric (into) mapping.

The proof can be found in $[5$, p. 82].

COROllary 2.3. If $F(X)$ is finite dimensional whenever $X$ is finite dimensional, $D F(X)$ is finite dimensional whenever $X$ is finite dimensional. If $F(X)$ is reflexive whenever $X$ is reflexive, $D F(X)$ is reflexive whenever $X$ is reflexive.

Definition 2.4. Let $F: B \rightarrow B$ be a functor. A subfunctor $G: B \rightarrow B$ of $F$ is a functor so that for each $X$ in $\boldsymbol{B}, G(X)$ is a closed subspace of $F(X)$; and for each $f: X \rightarrow Y$ in $B, G(f): G(X) \rightarrow G(Y)$ is the restriction of $F(f): F(X) \rightarrow F(Y)$ to $G(X)$.

LEMMA 2.5. For each functor $F: B \rightarrow \boldsymbol{B}, D F$ is naturally equivalent to a subfunctor $R$ of $F^{\#}$.

Proof. For each $A$ in $B$, let $R(A)$ be the subspace of $F\left(A^{*}\right)^{*}$ equivalent to $D F(A)$ by (2.2). Since the diagram

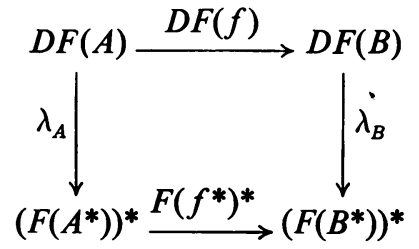

commutes for $f: A \rightarrow B$ in $B$, and each $y \in R(A)$ equals $\lambda_{A}(\theta)$ for a unique $\theta \in D F(A)$, $\left(F\left(f^{*}\right)\right)^{*}(y)=\left(\lambda_{B} \circ D F(f)\right) \theta$, an element in $R(B)$. This means $\left(F\left(f^{*}\right)\right)^{*}(R(A)) \subset R(B)$. 
Therefore, by defining $R(f)$ to be $\left(F\left(f^{*}\right)\right)^{*}$ restricted to $R(A)$ these assignments

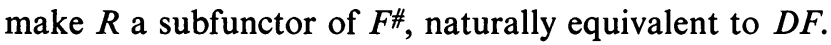

LemMa 2.6: Let $\tau: F \rightarrow G$ be a natural transformation. For each $A$ in $B$, let $\tau_{A}^{\#}$ be the morphism $\left.\left(\tau_{A^{*}}\right)^{*}:\left(G\left(A^{*}\right)\right)^{*} \rightarrow\left(F A^{*}\right)\right)^{*}$. The morphisms $\tau_{A}^{\#}$ generate a natural transformation $\tau^{\#:} G^{\#} \rightarrow F^{\#}$.

Proof. Let $f: X \rightarrow Y$ be in $B$. Since $\tau$ is a natural transformation the following diagram commutes.

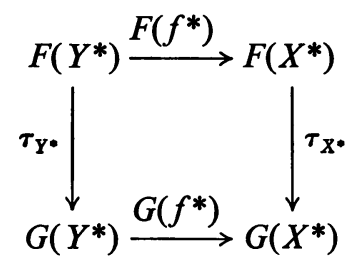

Hence the following diagram commutes.

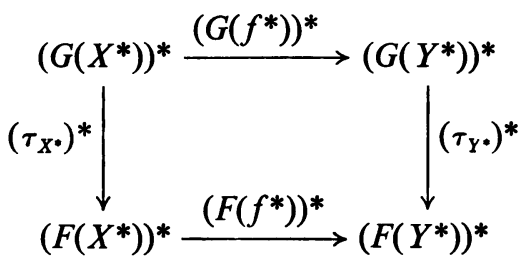

Since $\left|\tau_{A}^{\#}\right|=\left|\left(\tau_{A^{\circ}}\right)^{*}\right|=\left|\tau_{A^{*}}\right|,\left|\tau^{\#}\right|<\infty$ so that $\tau^{\#}$ is a natural transformation.

Definition 2.7. Let $\tau: F \rightarrow G$ be a natural transformation, $F^{\prime}$ a subfunctor of $F$, and $G^{\prime}$ a subfunctor of $G$. Then $F^{\prime}$ and $G^{\prime}$ are compatible subfunctors with respect to $\tau$ if for each $X$ in $B$, the restriction $r \tau_{X}$ of $\tau_{X}$ to $F^{\prime}(X) \subset F(X)$ maps $F^{\prime}(X)$ into $G^{\prime}(X)$.

LEMMA 2.8. The mappings $r \tau_{X}$ defined in (2.7) generate a natural transformation $r \tau: F^{\prime} \rightarrow G^{\prime}$

Proof. Let $f: X \rightarrow Y$ be in $B$. Since $r \tau_{X}, r \tau_{Y}, F^{\prime}(f)$, and $G^{\prime}(f)$ are the restriction of $\tau_{X}, \tau_{Y}, F(f)$, and $G(f)$ respectively, and $G(f) \circ \tau_{X}=\tau_{Y} \circ F(f)$, the morphism $G^{\prime}(f) \circ r \tau_{X}$ equals $r \tau_{Y} \circ F^{\prime}(f)$.

Proposition 2.9. Let $\tau: F \rightarrow G$ be a natural transformation and $\tau^{\#}: G^{\#} \rightarrow F^{\#}$ the generated transformation defined in (2.6). Then $D G$ and $D F$ are naturally equivalent to compatible subfunctors of $G^{\#}$ and $F^{\#}$ respectively (compatible with respect to $\tau^{\#)}$ ).

Proof. Let $R$ and $S$ be the subfunctors of $F^{\#}$ and $G^{\#}$ which by virtue of (2.5) are naturally equivalent to $D F$ and $D G$ respectively. Let $\lambda: D F \rightarrow F^{\#}$ and $\rho: D G$ 
$\rightarrow G^{\#}$ be these equivalences. Let $f: X \rightarrow Y$ be a morphism in $B$. Consider the following diagram.

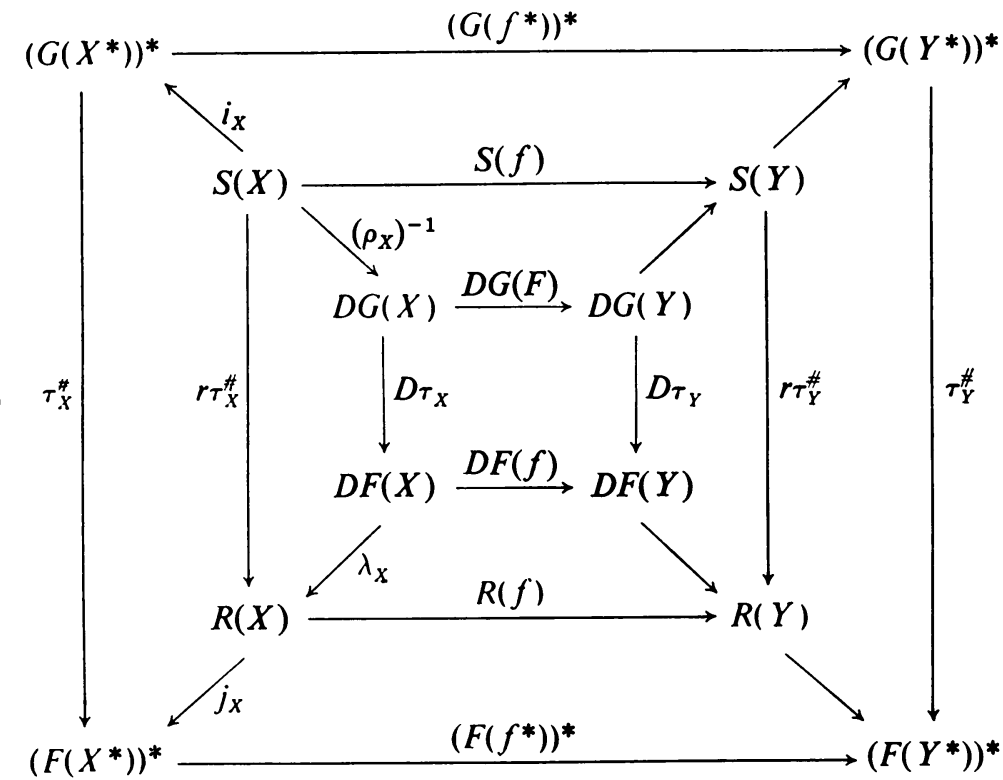

Define $r \tau_{X}^{\#}$ to be the morphism $\lambda_{X} \circ D \tau_{X} \circ\left(\rho_{X}\right)^{-1}$. It must be shown that $j_{X} \circ r \tau_{X}^{\#}$ $=\tau_{X}^{\#} \circ i_{X}$, where $i_{X}$ and $j_{X}$ are insertion maps. Let $s \in S(X)$. Then $s=\operatorname{Tr} \circ \theta_{X}$. for a unique $\theta \in D G(X)$. Also $\left(D \tau_{X}(\theta)\right)_{A}=\theta_{A} \circ \tau_{A}$ for each $A$ in $B$ and $D \tau_{X}(\theta) \in D F(X)$. Therefore, $r \tau_{X}^{\#}(S)$ in $R(X)$ is the element $\operatorname{Tr} \circ \theta_{X^{*}} \circ \tau_{X^{*}}: F\left(X^{*}\right) \rightarrow I$. Now consider $s=\operatorname{Tr} \circ \theta_{X^{*}}$ as an element of $\left(G\left(X^{*}\right)\right)^{*}$ via $i_{X}$. By definition $\tau_{X}^{\#}=\left(\tau_{X^{*}}\right)^{*}: G\left(X^{*}\right)^{*}$ $\rightarrow\left(F\left(X^{*}\right)\right)^{*}$. Therefore, $\tau_{X}^{\#}\left(\operatorname{Tr} \circ \theta_{X^{*}}\right)=\operatorname{Tr} \circ \theta_{X^{*}} \circ \tau_{X^{*}}$. Hence, $j_{X} \circ r \tau_{X}^{\#}=\tau_{X}^{\#} \circ i_{X}$ and $r \tau_{X}^{\#}$ is the restriction of $\tau_{X}^{\#}$ to $S(X)$. Similarly $r \tau_{Y}^{\#}$ is the restriction of $\tau_{Y}^{\#}$ to $S(Y)$.

Proposition 2.11. If $\tau: F \rightarrow G$ is a compact natural transformation, then $\tau^{\#:} G^{\#} \rightarrow F^{\#}$ is also.

Proof. Let $f: X \rightarrow Y$ be compact. It must be shown that any one of the equal morphisms

$$
\left(\tau_{Y^{*}}\right)^{*} \circ\left(G\left(f^{*}\right)\right)^{*}=\tau_{Y}^{\#} \circ G^{\#}(f)=F^{\#}(f) \circ \tau_{X}^{\#}=\left(F\left(f^{*}\right)\right)^{*} \circ\left(\tau_{X^{*}}\right)^{*}
$$

is compact. Since $f^{*}: Y^{*} \rightarrow X^{*}$ is compact $[1$, p. 485] and $\tau$ is a compact transformation, $G\left(f^{*}\right) \circ \tau_{Y^{*}}$ is compact. Hence $\left(G\left(f^{*}\right) \circ \tau_{Y^{*}}\right)^{*}=\left(\tau_{Y^{*}}\right)^{*} \circ\left(G\left(f^{*}\right)\right)^{*}$ is compact.

LemMA 2.12. Let $\tau: F \rightarrow G$ be a natural transformation, $F^{\prime}$ and $G^{\prime}$ compatible subfunctors with respect to $\tau$ of $F$ and $G$, and $r \tau: F^{\prime} \rightarrow G^{\prime}$ the transformation given by (2.8). If $\tau$ is a compact transformation so is $r \tau: F^{\prime} \rightarrow G^{\prime}$. 
Proof. Let $f: X \rightarrow Y$ be a compact mapping. The morphism $G^{\prime}(f) \circ r \tau_{X}$ is the restriction of the morphism $G(f) \circ \tau_{X}$ to $F^{\prime}(X)$. Let $S^{\prime}$ be the closed unit ball of $F^{\prime}(X)$ and $S$ the closed unit ball of $F(X)$. It suffices to show $\left(G^{\prime}(f) \circ r \tau_{X}\right)\left(S^{\prime}\right)$ is sequentially compact in $G^{\prime}(Y)$ (see [1, p. 22]). Since $S^{\prime} \subset S$ and $\left(G^{\prime}(f) \circ r \tau_{X}\right)\left(S^{\prime}\right)$ $=\left(G(f) \circ \tau_{X}\right)\left(S^{\prime}\right) \subset\left(G(f) \circ \tau_{X}\right)(S)$, every sequence of points in $\left(G\left(f^{\prime}\right) \circ r \tau_{X}\right)\left(S^{\prime}\right)$ is a sequence in $\left(G(f) \circ \tau_{X}\right)(S)$. Since $\left(G(f) \circ \tau_{X}\right)(S)$ is sequentially compact, every sequence of points in $\left(F\left(f^{\prime}\right) \circ r \tau_{X}\right)\left(S^{\prime}\right)$ has a subsequence converging to a point of $G(Y)$. However, since $G^{\prime}(Y)$ is closed in $G(Y)$, this point is in $G^{\prime}(Y)$ and $\left(G\left(f^{\prime}\right) \circ \gamma \tau_{X}\right)\left(S^{\prime}\right)$ is sequentially compact.

LEMMA 2.13. Let $\tau: F \rightarrow H$ be the composition of the natural transformation $\eta: F \rightarrow G$ and $\theta: G \rightarrow H$. If $\theta$ or $\eta$ is compact, $\tau$ is compact.

Proof. It is a direct consequence of the fact that the composition of any operator with a compact operator is compact (see [1, p. 486]).

THEOREM 2.14. If $\tau: F \rightarrow G$ is a compact transformation, then $D \tau: D G \rightarrow D F$ is a compact natural transformation.

Proof. Since $\tau: F \rightarrow G$ is compact, by (2.11) $\tau^{\#}: F^{\#} \rightarrow G^{\#}$ is compact. By (2.9) $D G$ and $D F$ are naturally equivalent to compatible subfunctors $S$ and $R$ of $G^{\#}$ and $F^{\#}$. By (2.12), the transformation $r \tau^{\#}: S \rightarrow R$ is compact. Referring to diagram (2.10), $D \tau_{X}=\left(\lambda_{X}\right)^{-1} \circ r \tau_{X}^{\#} \circ \rho_{X}$ for each $X$ in $B$, or $D \tau=\lambda^{-1} \circ r \tau^{\#} \circ \rho$. By (2.13), $D \tau$ is compact.

THEOREM 2.15. If $\tau: F \rightarrow G$ is a weakly compact natural transformation, then $D \tau: D G \rightarrow D F$ is a weakly compact natural transformation.

Proof. By similar proofs, it can be shown that with the words "weakly compact" inserted for the word "compact" in (2.11), (2.12), and (2.13), the corresponding statements are true. Using these altered statements, the proof of this theorem is similar to the proof of (2.14).

THEOREM 2.16. If $\tau: F \rightarrow G$ is an epimorphic natural transformation, then $D \tau: D G \rightarrow D F$ is a monomorphic natural transformation.

Proof. Let $f: A \rightarrow B$ be a monomorphism. Then $f^{*}: B^{*} \rightarrow A^{*}$ is an epimorphism. Hence, $\tau_{A^{*}} \circ F\left(f^{*}\right): F\left(B^{*}\right) \rightarrow G\left(A^{*}\right)$ is an epimorphism since $\tau$ is epimorphic. Therefore

$$
\left(\tau_{A^{*}} \circ F\left(f^{*}\right)\right)^{*}=\left(F\left(f^{*}\right)\right)^{*} \circ\left(\tau_{A^{*}}\right)^{*}=F^{\#}(f) \circ \tau_{A}^{\#}=\tau^{\#}(f)
$$

is a monomorphism, or $\tau^{\#}: G^{\#} \rightarrow F^{\#}$ is monomorphic. Using diagram (2.10), it can be seen that $D F(f) \circ D \tau_{X}$ is a monomorphism. Hence $D \tau$ is a monomorphism.

THeORem 2.17. Let $F: B \rightarrow B$ be a functor. Then

(1) if $F$ is compact, $D F$ is compact;

(2) if $F$ is weakly compact, DF is weakly compact; and

(3) if $F$ is epimorphic, DF is monomorphic. 
Proof. If $\iota_{F}: F \rightarrow F$ is the identity transformation, then $D \iota_{F}=\iota_{F}$ (naturally equivalent). Therefore if $\iota_{F}$ is compact, weakly compact, or epimorphic, by (2.14), (2.15), and (2.16), $D \iota_{F}=\iota_{F}$ is respectively compact, weakly compact, or monomorphic.

Results (2.14) through (2.17) have been proved independently in a similar way by Evans in [2].

3. Characterizations of compact functors. In this section, necessary and sufficient conditions will be given for the functors $\Sigma_{X}$ and $\Omega_{X}$ to be compact. More generally, conditions will be given to insure certain functors $F: \boldsymbol{B} \rightarrow \boldsymbol{B}$ are compact.

Similar to (2.1), a functor $F: \boldsymbol{B} \rightarrow \boldsymbol{B}$ has finite rank (or is a finite rank functor) if whenever a morphism $f: A \rightarrow B$ has finite rank $(\operatorname{dim} f(A)$ is finite), $F(f): F(A) \rightarrow$ $F(B)$ has finite rank.

The next lemma is easy to prove.

LEMMA 3.1. A functor $F: B \rightarrow B$ has finite rank if and only if $F(X)$ is finite dimensional whenever $X$ is finite dimensional.

A morphism $f: A \rightarrow B$ in $B$ is Fredholm if $\operatorname{Ker} f=f^{-1}(0)$ and Coker $f=Y \mid f(X)$ are finite dimensional.

The proof of the following lemma can be found in [6, p. 120].

Lemma 3.2. Let $X$ and $Y$ be in $B$. If $f \in B(X, Y)$ and there exist $h$ and $h^{\prime}$ in $B(Y, X)$ such that $h \circ f-i_{X}$ and $f \circ h^{\prime}-i_{Y}$ are compact, then $f$ is Fredholm. Conversely, if $f$ is a Fredholm operator, then there exists a $g$ in $B(Y, X)$ so that $g \circ f-i_{X}$ and $f \circ g-i_{Y}$ have finite rank and hence are compact.

Proposition 3.3. A functor $F: B \rightarrow B$ is a Fredholm functor (see (2.1)) if and only if it has finite rank.

Proof. Let $A$ be a finite-dimensional Banach space. Then $0: A \rightarrow A$ (the zero morphism) is a Fredholm operator. Hence $F(0)=0: F(A) \rightarrow F(A)$ is Fredholm, which implies $\operatorname{Ker} F(0)=F(A)$ is finite dimensional.

Conversely, let $F$ have finite rank and let $f: A \rightarrow B$ be a Fredholm operator. Using (3.2), there is a $g: B \rightarrow A$ in $B$ such that $f \circ g-i_{B}$ and $g \circ f-i_{A}$ have finite rank. Hence $F(f) \circ F(g)-i_{F(B)}$ and $F(g) \circ F(f)-i_{F(A)}$, have finite rank and therefore are compact. By (3.2) $F(f)$ is a Fredholm operator.

Corollary 3.4. If $F: B \rightarrow B$ is Fredholm, so is $D F: B \rightarrow \boldsymbol{B}$.

Proof. If $F$ has finite rank, so does $D F$ by (3.1) and (2.3).

LEMMA 3.5. If $F: B \rightarrow B$ is a compact functor, then $F$ has finite rank and hence is $a$ Fredholm functor. 
Proof. $A$ is a finite-dimensional Banach space if and only if $i_{A}: A \rightarrow A$ is compact. If $A$ is finite dimensional, by assumption $i_{F(A)}: F(A) \rightarrow F(A)$ is compact so that $F(A)$ is finite dimensional. The result follows from (3.1).

COROLlaRY 3.6. If $\Sigma_{X}$ is a compact functor, $X$ must be finite dimensional.

Proof. By (3.5), if $A$ is a finite-dimensional Banach space, then $X \widehat{\otimes} A$ must be finite dimensional. In particular, $X \hat{\otimes} I$ equivalent to $X$ must have finite dimension.

Let $E$ be a set. The notation $l_{A}(E)$ for $A$ in $B$ represents the Banach space of all functions $t: E \rightarrow A$ such that the "norm" of $t$ given by $|t|=\sum_{e \in E}|t(e)|$ is finite. In particular, if $E=N=\{1,2, \ldots, n\}, l_{A}(N)$ is the set of all $n$-tuples $a=\left(a_{1}, \ldots, a_{n}\right)$ made up of elements of $A$ with norm given by $|a|=\sum_{i=1}^{n}\left|a_{i}\right|$. Define the functor $l_{n}: B \rightarrow B$ by $l_{n}(A)=l_{A}(N)$.

Proposition 3.7. For each integer $n, l_{n}: \boldsymbol{B} \rightarrow \boldsymbol{B}$ is a compact functor.

Proof. Let $f: A \rightarrow B$ be a compact mapping. Denote by $\left(a_{1 j}, \ldots, a_{n j}\right)$ the $j$ th term $\left(a_{1}, \ldots, a_{n}\right)_{j}$ of a sequence of elements of $l_{n}(A)$. Then $l_{n}(f)\left(\left(a_{1 j}, \ldots, a_{n j}\right)\right)$ is the element $\left(f\left(a_{1 j}\right), \ldots, f\left(a_{n j}\right)\right)$ of $l_{n}(B)$. It suffices to show that if $\left(a_{1 j}, \ldots, a_{n j}\right)$ for $j=1,2, \ldots$ is a sequence in $l_{n}(A)$ so that, for each $j, \sum_{i=1}^{n}\left|a_{i j}\right|=1$, then $\left(f\left(a_{1 j}\right), \ldots\right.$, $\left.f\left(a_{n j}\right)\right)$ for $j=1,2, \ldots$ has a convergent subsequence. This is done by extracting first a subsequence $f\left(a_{1 j_{k}}\right)$ from $f\left(a_{1 j}\right)$ converging to a point $b_{1}$ of $B$, and then extracting from $f\left(a_{2 j_{k}}\right)$ a subsequence converging to a point $b_{2}$ of $B$, and so on. This can be done since $f$ is compact. Eventually a subsequence of $\left(f\left(a_{1 j}\right), f\left(a_{2 j}\right)\right.$, $\left.\ldots, f\left(a_{n j}\right)\right)$ is obtained converging to the point $\left(b_{1}, b_{2}, \ldots, b_{n}\right)$ of $l_{n}(B)$.

PROPOSITION 3.8. The functor $\Sigma_{l_{I}(N)}$ is a compact functor.

Proof. A special case of a result due to Grothendieck [3, p. 59] says that the functors $\Sigma_{l_{I}(N)}$ and $l_{n}$ are naturally equivalent where $N=(1,2, \ldots, n)$. Let the equivalence be $\tau$ and let $f: A \rightarrow B$ be a compact mapping. By naturality, the following diagram commutes.

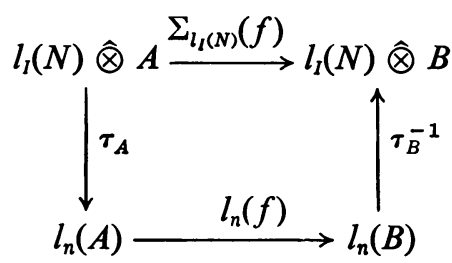

By (3.7), $l_{n}(f)$ is compact. Therefore $\Sigma_{l_{l}(N)}$ is compact.

Proposition 3.9. If $X$ is a finite-dimensional Banach space, then $\Sigma_{X}: B \rightarrow B$ is $a$ compact functor. 
Proof. Let the dimension of $X$ be $n$. Since $l_{I}(N)$ has dimension $n, X$ and $l_{I}(N)$ are isomorphic by an isomorphism $f$. If $g: A \rightarrow B$ is in $B$, the following diagram commutes.

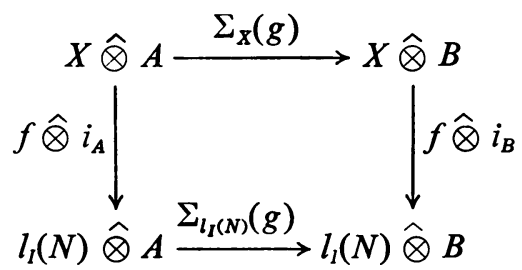

If $g$ is compact, then by (3.8) $\Sigma_{l_{I}(N)}(g)$ is compact. Therefore $\Sigma_{X}(g)$ is compact.

The following theorem summarizes the above discussion.

THEOREM 3.10. The following statements are equivalent.

(1) $X$ is a finite-dimensional Banach space.

(2) $\Sigma_{X}: B \rightarrow B$ is a compact functor.

(3) $\Sigma_{X}: B \rightarrow B$ is a Fredholm functor.

(4) $\Omega_{X}: \boldsymbol{B} \rightarrow \boldsymbol{B}$ is a compact functor.

(5) $\Omega_{X}: B \rightarrow B$ is a Fredholm functor.

The question remains whether each finite rank functor is a compact functor. The following discussion shows that the answer is yes in all known cases.

Definition 3.11. A Banach space $X$ is said to satisfy the approximation property if for each $\varepsilon>0$ and for each relatively compact subset $C$ of $X$, there exists a map $f: X \rightarrow X$ of finite rank so that $|f(x)-x|<\varepsilon$ for all $x$ in $C$.

Note. No Banach space is known that does not satisfy (3.11). See [3, p. 135] and $[9$, p. 521].

The following lemma is contained in a result by Grothendieck [3, p. 168].

LeMma 3.12. Let $X$ be a Banach space that satisfies (3.11). Then for any Banach space $Y$, for any compact mapping $f: Y \rightarrow X$, there exists for each $\varepsilon>0$ a mapping $f_{\varepsilon}: Y \rightarrow X$ of finite rank such that $\left|f-f_{\varepsilon}\right|<\varepsilon$.

Proposition 3.13. Let $\boldsymbol{B}^{\prime}$ denote the full subcategory of $\boldsymbol{B}$ consisting of Banach spaces which satisfy the approximation property. Then a functor $F: \boldsymbol{B}^{\prime} \rightarrow \boldsymbol{B}^{\prime}$ is compact if and only if $F$ is a finite rank functor.

Proof. Necessity follows from (3.5). To prove the converse, let $f: A \rightarrow B$ be a compact operator in $\boldsymbol{B}^{\prime}$. By (3.12), there exists a sequence $f_{n}: A \rightarrow B$ of operators with finite-dimensional range in $B$ converging to $f$ in the norm of $B(A, B)$. By the hypothesis, $F\left(f_{n}\right): F(A) \rightarrow F(B)$ are operators of finite rank and hence are compact. Since $\left|F\left(f_{n}\right)-F(f)\right| \leqq\left|f_{n}-f\right|$ for $n=1,2, \ldots$, the sequence $F\left(f_{n}\right)$ converges to $F(f)$ in the norm of $\boldsymbol{B}(F(A), F(B))$. Hence $F(f)$ is compact [1, p. 486]. Q.E.D.

An example of a functor satisfying (3.13) is the functor $l_{n}$. Since for any Banach 
space $X$ of dimension $n, \Sigma_{X} A$ and $\Omega_{X} A$ are isomorphic to $l_{n} A$ (see [9, p. 522]), $\Sigma_{X}$ and $\Omega_{X}$ also satisfy (3.13) if $X$ is of finite dimension.

THEOREM 3.14. Let $f: A \rightarrow B$ be any compact mapping with $B$ satisfying the approximation property. If $F: B \rightarrow B$ is a functor, then $F(f): F(A) \rightarrow F(B)$ is compact if and only if $F$ is a finite rank functor.

Proof. The proof is identical to that of (3.13).

The following lemma is similar to (3.12) and is contained in a result by Grothendieck [3, p. 168].

LEMMA 3.15. If $Y$ is in $\boldsymbol{B}$ so that $Y^{*}$ satisfies the approximation property, then for any Banach space $X$, for any compact mapping $f: Y \rightarrow X$, there exists for each $\varepsilon>0$ a mapping $f_{\varepsilon}: Y \rightarrow X$ in $\boldsymbol{B}$ of finite rank so that $\left|f_{\varepsilon}-f\right|<\varepsilon$.

THEOREM 3.16. Let $f: Y \rightarrow X$ be any compact map with $Y^{*}$ satisfying the approximation property. If $F: B \rightarrow B$ is a functor, then $F(f)$ is compact if and only if $F$ is a finite rank functor.

LEMMA 3.17. Let $h: C \rightarrow A$ be a normal epimorphism (an epimorphism with the induced map $C / \operatorname{Ker} h \rightarrow A$ isometric) and $g: B \rightarrow D$ an isometric (into) map in $B$. Then

(1) $f: A \rightarrow B$ is compact if and only if $f \circ h: C \rightarrow B$ is compact, and

(2) $f: A \rightarrow B$ is compact if and only if $g \circ f: A \rightarrow D$ is compact.

Proposition 3.18. Let $F: B \rightarrow B$ be a functor.

(1) If $F$ is a finite rank functor and a normal epimorphic functor (preserves normal epimorphism), then $F$ is compact.

(2) If $F$ is a finite rank functor and preserves normal monomorphisms, then $F$ is compact.

Proof. (1) Let $f: A \rightarrow B$ be a compact map. Consider the space $l_{I}(S)$, where $S$ is the unit ball of $A$. Then the map $h: l_{l}(S) \rightarrow A$ given by $h(t)=\sum_{s \in S} t(s) s$ is a normal epimorphism. The operator $f \circ h: l_{l}(S) \rightarrow B$ is compact. According to Grothendieck [3, p. 185], the space $\left(l_{I}(S)\right)^{*}$ satisfies the approximation property. By (3.16), $F(f \circ h)=F(f) \circ F(h)$ is compact. Since $F(h)$ is a normal epimorphism, by (3.17) $F(f)$ is compact.

(2) Again let $f: A \rightarrow B$ be a compact map. Let $S^{*}$ be the unit ball of $B^{*}$. The dual of $l_{I}\left(S^{*}\right)$ is the space $l_{I}^{\infty}\left(S^{*}\right)$, the Banach space of all functions $x: S^{*} \rightarrow I$ such that $|x|=\sup _{s^{*} \in S^{*}}\left|x\left(S^{*}\right)\right|$ is finite. According to Grothendieck [3, p. 185], $l_{I}^{\infty}\left(S^{*}\right)$ satisfies the approximation property. The map $g: B \rightarrow l_{I}^{\infty}\left(S^{*}\right)$ given by $g(b)=x_{b}: S^{*} \rightarrow I$, where $x_{b}\left(s^{*}\right)=s^{*}(b)$ is an isometric map. The map $g \circ f$ is compact. By (3.14), $F(g \circ f)=F(g) \circ F(f)$ is a compact map. Since $F(g)$ is an isometric map, $F(f)$ is compact by (3.17).

The author wishes to express his thanks to Professor Kung-Wei Yang for his help in preparing this paper. 


\section{REFERENCES}

1. N. Dunford and J. T. Schwartz, Linear operators. I: General theory, Pure and Appl. Math., vol. 7, Interscience, New York, 1958. MR 22 \#8302.

2. Noel D. Evans, Functors in categories of Banach spaces, Unpublished Doctoral Dissertation, University of Texas, Austin, 1968.

3. A. Grothendieck, Produits tensoriels topologiques et espaces nucléaires, Mem. Amer. Math. Soc. No. 16 (1955). MR 17, 763.

4. B. Mitchell, Theory of categories, Pure and Appl. Math., vol. 17, Academic Press, New York, 1965. MR 34 \#2647.

5. B. S. Mitjagin and A. S. Svarc, Functors in categories of Banach spaces, Uspehi Mat. Nauk 19 (1964), no. 2 (116), 65-130= Russian Math. Surveys 19 (1964), no. 2, 65-127. MR 29 \#3866.

6. R. S. Palais, Seminar of the Atiyah-Singer index theorem, Ann. of Math. Studies, no. 57, Princeton Univ. Press, Princeton, N. J., 1965. MR 33 \#6649.

7. H. H. Schaefer, Topological vector spaces, Macmillan, New York, 1966. MR 33 \#1689.

8. R. Schatten, On the direct product of Banach spaces, Trans. Amer. Math. Soc. 53 (1943), 195-217. MR 4, 161.

9. F. Treves, Topological vector spaces, distributions, and kernels, Academic Press, New York, 1967. MR 37 \#726.

WeStern Michigan University, Kalamazoo, Michigan 49001 